\title{
Constrained Log-Likelihood-Based Semi-supervised Linear Discriminant Analysis
}

\author{
Marco $\operatorname{Loog}^{1}$ and Are C. Jensen ${ }^{2}$ \\ 1 Pattern Recognition Laboratory, Delft University of Technology \\ Delft, The Netherlands \\ prlab.tudelft.nl \\ 2 Department of Informatics, University of Oslo \\ Oslo, Norway
}

\begin{abstract}
A novel approach to semi-supervised learning for classical Fisher linear discriminant analysis is presented. It formulates the problem in terms of a constrained log-likelihood approach, where the semisupervision comes in through the constraints. These constraints encode that the parameters in linear discriminant analysis fulfill particular relations involving label-dependent and label-independent quantities. In this way, the latter type of parameters, which can be estimated based on unlabeled data, impose constraints on the former. The former parameters are the class-conditional means and the average within-class covariance matrix, which are the parameters of interest in linear discriminant analysis. The constraints lead to a reduction in variability of the label-dependent estimates, resulting in a potential improvement of the semi-supervised linear discriminant over that of its regular supervised counterpart. We state upfront that some of the key insights in this contribution have been published previously in a workshop paper by the first author. The major contribution in this work is the basic observation that a semi-supervised linear discriminant analysis can be formulated in terms of a principled log-likelihood approach, where the previous solution employed an ad hoc procedure. With the current contribution, we move yet another step closer to a proper formulation of a semi-supervised version of this classical technique.
\end{abstract}

\section{Introduction}

Supervised learning aims to learn from examples. That is, given a limited number of instances of a particular input-output relation, its goal is to generalize this relationship to new and unseen data in order to enable the prediction of the associated output given new input. Specifically, supervised classification aims to infer an unknown feature vector-class label relation from a finite, potentially small, number of input feature vectors and their associated, desired output class labels. Now, an elementary question is whether and, if so, how the availability of additional unlabeled data can significantly improve the training of such classifier. This is what constitutes the problem of semi-supervised classification or, generally, semi-supervised learning 322 . 
The hope or, rather, belief is that semi-supervision can bring enormous progress to many scientific and application areas in which classification problems play a key role, simply by exploiting the often enormous amounts of unlabeled data available (think computer vision, text mining, retrieval, medical diagnostics, but also social sciences, psychometrics, econometrics, etc.). The matter of the fact, however, is that up to now semi-supervised methods have not been widely accepted outside of the realms of computer science, being little used in other domains. Part of the reason for this may be that current methods offer no performance guarantees [20] and often deteriorate in the light of large amounts of unlabeled samples 4518.

References [10] and 11] identify as main reason for the frequent failure of semi-supervision that current semi-supervised approaches typically rely on assumptions extraneous to the classifier being considered. A similar point has been raised in [13. Indeed, the main current approaches to semi-supervised learning stress the need for presuppositions such as the cluster assumption: points from the same class cluster, the smoothness assumption: neighboring point have the same label, the assumption of low density separation: the decision boundary is located in low density areas, and the like [322]. Given a particular assumption holds, one is able to extract relevant information not only from the labeled, but especially from the unlabeled examples. While it is undeniably true that having more precise knowledge on the distribution of data could, or even should, help in training a better classifier, in many real-world settings it may be questionable if one can at all check if such conditions are indeed met. Moreover, as soon as these additional model assumptions do not fit the data, there obviously is the real risk that adding unlabeled data actually leads to a severe deterioration of classification performance 45/101118. Note that this is in contrast with the supervised setting, where most classifiers, generative or not, are capable of handling mismatched data assumptions rather well, in the sense that adding more training data generally improves the performance of the classifier.

This work continues in the spirit of the earlier research presented in [10] and [1]. Reference [10] introduces a semi-supervised version of the simple, at times still topical [919], nearest mean classifier (NMC, 16]). It suggests to exploit known relationships between the class means and the label-independent overall mean. Enforcing these constraints during semi-supervision, yields labeldependent estimates that have smaller expected deviation from the true parameter value, which, in turn, leads to reduced classification errors. In fact, despite its simplicity, semi-supervised NMC in some cases provides error rates that are competitive with state-of-the-art methods (compare [10] and [3]). Where [10] presents a straightforward way to enforce labeled-unlabeled constraints merely involving class means and overall means, [1] shows how to deal with a known constraint on the average within-class covariance matrix as well. The constraint is relevant to linear discriminant analysis (LDA) but more difficult to deal with. Results in [11] show the overall good performance of semi-supervised LDA, not only when compared to standard supervised LDA setting but also in the light 
of earlier approaches to semi-supervised LDA, which often show detrimental performance with large amounts of unlabeled samples indeed.

Where the earlier approach provide an ad hoc ways to enforce the constraints, this paper casts the problem into a principled log-likelihood framework, basically proposing to optimize the regular likelihood underlying LDA under the constraint proposed in [11.

After the next section, which presents a brief overview of related work, Section 3 briefly recapitulates the relevant details of the approach presented in [10. The main focus in that section will, however, be on semi-supervised LDA as presented in [11] and the better founded approach to semi-supervision through the loglikelihood. Section 4 offers an experiment by means of which we try to get an initial impression about how different the new principled approach is from the earlier ad hoc technique. In addition, it reports on the results obtained. Section 5 wraps up the work with a discussion and conclusion.

\section{Related Work}

There are few works that focus on semi-supervised LDA. Most relevant contributions come from statistics and have been published mainly in the 1960s and 1970s. Reference [8] suggests to maximize the likelihood over all permutations of possible labelings of unlabeled objects. A computationally more feasible approach is proposed by McLachlan [1415, which follow an iterative procedure. Firstly, the linear discriminant is trained on the labeled data only and used to label all unlabeled instances. Using the now-labeled data, the classifier is retrained and employed to relabel the initially unlabeled data. This process of relabeling originally unlabeled data is repeated until none of the samples changes label.

The above approach to semi-supervised learning is basically a form of socalled self-training or self-learning, which has been suggested in different guises [14 17/21]. This iterative method also relates directly to the well-known approach to semi-supervision based on expectation maximization (see [18] and the discussion papers related to [6]). We note that employing expectation maximization to infer the missing labels will in many cases also lead to worsened error rates, particularly if too many unlabeled examples are included.

Finally, we remark that there are also semi-supervised approach to LDA as a dimensionality reduction technique (as opposed to LDA as a classifier) but we refrain from reviewing these works here.

\section{Constrained Log-Likelihood-Based LDA}

\subsection{Semi-supervised NMC Basics}

The semi-supervised version of the (NMC) proposed in [10] is simple but has been proven to be effective notwithstanding. To start with, note that when employing 
a regular supervised NMC, the $K$ class means, $m_{i}$ with $i \in\{1, \ldots, K\}$, and the overall mean of the data, $m$, fulfill the linear constraint 7 ]

$$
m=\sum_{i=1}^{K} p_{i} m_{i}
$$

where $p_{i}$ is the prior of class $i$. Having additional unlabeled data, one can improve the estimate of $m$ because it does not depend on any labels. In this case, however, the constraint in Equation (11) will typically be violated. The core idea in [10] is that one can get improved estimates of the class means by adapting them such that the constraint is satisfied again. The solution chosen is to simply alter the $K$ sample class means $m_{i}$ by the same shift such that the new total sample mean $m^{\prime}=\sum_{i=1}^{K} p_{i} m_{i}^{\prime}$ of the shifted class means $m_{i}^{\prime}$ coincides with the total sample mean $\mu$. The total mean $m^{\prime}$ has been obtained using all data available. All in all, the following update of the class means is suggested

$$
m_{i}^{\prime}=m_{i}-\sum_{i=1}^{K} p_{i} m_{i}+\mu
$$

\subsection{Ad Hoc Semi-supervised LDA}

For LDA, next to Equation (1), an additional known constraint equates the sum of the estimates of the between-class covariance matrix $\mathbf{B}$ and within-class covariance $\mathbf{W}$ to the total covariance over all data $\mathbf{T}$ (cf. [7]). That is

$$
\mathbf{T}=\mathbf{W}+\mathbf{B}
$$

where

$$
\mathbf{T}:=\frac{1}{N} \sum_{i=1}^{K} \sum_{j=1}^{N_{i}}\left(x_{i, j}-m\right)\left(x_{i, j}-m\right)^{\mathrm{t}},
$$

in which $x_{i, j}$ is the $j$ th feature vector from class $i, m$ is the estimated overall mean, $N_{i}$ is the number of samples from class $i$, and $N$ is the total number of samples. The remaining variables in the equation have the following definitions:

$$
\mathbf{W}:=\sum_{i=1}^{K} p_{i} \mathbf{C}_{i}
$$

where $\mathbf{C}_{i}$ is the sample covariance matrix for class $i$, and

$$
\mathbf{B}:=\sum_{i=1}^{K} p_{i}\left(m_{i}-m\right)\left(m_{i}-m\right)^{\mathrm{t}} .
$$

The parameters of interest are the class means $m_{i}$, the within-class covariance matrix $\mathbf{W}$, and the priors $p_{i}$. These parameters should be estimated from both 
labeled and unlabeled data under the constraints provided in Equations (11) and (3), in which the left hand side is fixed and determined by all data available.

Now, denote the estimated total mean based on all the data by $\mu$, as in Subsection 3.1 and let the corresponding total covariance matrix be denoted by $\boldsymbol{\Theta}$. The corresponding mean $m$ and covariance matrix $\mathbf{T}$ are based merely on the labeled data. Reference [11] now suggests the following easy and effective solution in order to meet the constraints. To start with, transform every labeled datum $x$ as follows:

$$
x \leftarrow \boldsymbol{\Theta}^{\frac{1}{2}} \mathbf{T}^{-\frac{1}{2}}(x-m)+\mu .
$$

The transformation sees to it that the overall mean and covariance statistics of the labeled data match the respective statistics as measured on all data. That is, on the transformed data, the corresponding $m$ and $\mathbf{T}$ equal $\mu$ and $\boldsymbol{\Theta}$, respectively. The next step is to simply train a regular LDA on this transformed training data, providing the semi-supervised estimates for $m_{i}$ and $\mathbf{W}$. By means of Equation (6), the corresponding $\mathbf{B}$ in the transformed space can be determined. By construction, one has

$$
\mu=\sum_{i=1}^{K} p_{i} m_{i}
$$

and

$$
\Theta=\mathbf{W}+\mathbf{B} .
$$

As the transformation applied is affine, we can actually directly estimate the $m_{i} \mathrm{~s}$ and the $\mathbf{W}$ in the original space. Given the class means $m_{i}^{\prime}$ and the within-class covariance matrix $\mathbf{W}^{\prime}$ determined on the untransformed labeled data only, the following holds:

$$
\begin{aligned}
& m_{i}=\boldsymbol{\Theta}^{\frac{1}{2}} \mathbf{T}^{-\frac{1}{2}}\left(m_{i}^{\prime}-m\right)+\mu \\
& \mathbf{W}=\mathbf{T}^{-\frac{1}{2}} \boldsymbol{\Theta}^{\frac{1}{2}} \mathbf{W}^{\prime} \boldsymbol{\Theta}^{\frac{1}{2}} \mathbf{T}^{-\frac{1}{2}} .
\end{aligned}
$$

This expresses the $m_{i} \mathrm{~s}$ and $\mathbf{W}$ in terms of first and second order moment statistics in the original space.

Experiments have demonstrated that this approach outperforms standard LDA in most cases. What might be even more important, however, is that it is better behaved than the self-learning and EM-type of approaches, not showing the extreme detrimental behavior the latter methods can display at times.

\subsection{Constrained Log-Likelihood-Based LDA}

A basic problem with the foregoing solution is that it is unclear in which way it can be considered optimal. It delivers the necessary means and covariance matrices such that the constraints are satisfied but there are infinitely many solutions fulfilling the same constraints. Some of these can be easily constructed by assigning arbitrary labels to the unlabeled data and training a standard LDA. As can be checked easily, the parameters estimated in this way will necessarily 
satisfy the constraints given in Equations (11) and (3). The simplicity of the solution from [1] may be appealing but there really seems to be little reason to prefer it over any of the others. There is, however, a solution to this matter that, by now, should not come as a surprise (see [11]).

The maximum likelihood solution typical employed for LDA, finds the parameters $p_{i}, m_{i}$, and $\mathbf{W}$ by optimizing the log-likelihood of the complete model (see, for instance, [16]). In the paper's notation, for a given labeled data set, this $\log$-likelihood $L$ can be expressed as follows:

$$
\begin{aligned}
& L\left(p_{i}, m_{i} \mathbf{W}\right)= \\
& \sum_{i=1}^{K} \sum_{j=1}^{N_{i}}\left(\log p_{i}-\frac{d}{2} \log 2 \pi-\frac{1}{2} \log |\mathbf{W}|-\frac{1}{2}\left(x_{i, j}-m_{i}\right) \mathbf{W}^{-1}\left(x_{i, j}-m_{i}\right)^{\mathrm{t}}\right) .
\end{aligned}
$$

Maximizing this expression directly leads to the standard sample estimates for the class priors, the class means, and the average within-class covariance matrix, provided all $p_{i}$ s are positive and add up to 1 . Now, next to the constraint on the priors, as an elegant solution to the semi-supervised estimation problem, the constraints in Equations (11) and (3), which rely on unlabeled data as well, could be imposed in addition.

This choice is much more attractive than the ad hoc solution from [11, in the sense that there is a clear optimality criterion at the basis of this semisupervised solution to LDA. In addition, it is based on the log-loss that is at the basis of LDA in the first place. The price we pay is that we run into a more complicated optimization problem as the constrained log-likelihood formulation does not allow for a closed-form solution nor is it convex.

\section{Experiment and Results}

A small experiment was conducted, basically to get an impression of how our new approach to semi-supervision compares to the earlier suggestion from [1]. Experiments with standard supervised LDA are included as well, for comparison but also to remind us that we are still not there: occasionally supervised LDA will still outperform its semi-supervised variants.

The constrained log-likelihood is optimized by means of a Hessian-corrected gradient ascent on the constrained means $m_{i}$ (Newton's method). In every iteration, given the updated means, the new within-class covariance matrix $\mathbf{W}$ can be determined and we can check if it fails to be positive definite. If this happens, we decrease the step size of the ascend and reevaluate $\mathbf{W}$. A similar action is taken in case the log-likelihood decreases. In the experiments, two different starting points satisfying the various constraints are employed. The first one, which is referred to as $\alpha$, initializes all class means by the total data mean $\mu$ and the

\footnotetext{
${ }^{1}$ Maybe, though, one might be able to demonstrate that the data transformation as suggested in Subsection 3.2 is the one that deforms the original data in some sort of minimal way. Up to now, however, we have been unsuccessful in showing this.
} 
within covariance matrix by the total covariance $\mathbf{T}$. The second starting point is provided by the ad hoc solution from Subsection 3.2 as presented in [1], which is referred to as $\Omega$.

Table 1. Some basic properties of the fourteen real-world data sets

\begin{tabular}{|c|c|c|c|c|c|c|}
\hline data set & $\begin{array}{c}\text { number of } \\
\text { objects }\end{array}$ & $\begin{array}{c}\text { dimensionality } \\
\text { (original) }\end{array}$ & $\begin{array}{c}\text { dimensionality } \\
\text { (after PCA) }\end{array}$ & $\begin{array}{c}\text { number of } \\
\text { class }\end{array}$ & $\begin{array}{l}\text { smallest } \\
\text { class size }\end{array}$ & $\mid \begin{array}{c}\text { largest } \\
\text { class size }\end{array}$ \\
\hline glass & 214 & 9 & 6 & 6 & 9 & 76 \\
\hline haberman & 306 & 3 & 3 & 2 & 81 & 225 \\
\hline ionosphere & 351 & 33 & 30 & 2 & 126 & 225 \\
\hline iris & 150 & 4 & 3 & 3 & 50 & 50 \\
\hline parkinsons & 195 & 22 & 3 & 2 & 48 & 147 \\
\hline pendigits & 10992 & 16 & 13 & 10 & 1055 & 1144 \\
\hline pima & 768 & 8 & 5 & 2 & 268 & 500 \\
\hline sat & 6435 & 36 & 16 & 6 & 626 & 1533 \\
\hline segmentation & 210 & 19 & 5 & 7 & 30 & 30 \\
\hline sonar & 208 & 60 & 29 & 2 & 97 & 111 \\
\hline spambase & 4601 & 57 & 2 & 2 & 1813 & 2788 \\
\hline transfusion & 748 & 3 & 3 & 2 & 178 & 570 \\
\hline vowel & 990 & 10 & 9 & 11 & 90 & 90 \\
\hline wdbc & 569 & 30 & 2 & 2 & 212 & 357 \\
\hline
\end{tabular}

For the experiments, fourteen real-world data sets are taken from the UCI Machine Learning Repository [1. The data sets used together with some specifications can be found in Table 1]. To avoid any problems with singular total covariance matrices, the dimensionality of all data sets is initially reduced using PCA so to retain a fraction of 0.99 of the total variance. The data dimensionalities after PCA can be found in column four of the table.

The largest effect of semi-supervision may be expected when the labeled training set is small. Training set sizes are therefore set to equal the dimensionality plus the number of classes, which makes sure that the within-class covariance is still invertible. The remainder of the data set is both used as unlabeled data for the semi-supervised learners and as test set, meaning that we are in a transductive setting. This random split of data is repeated 10 times for every experiment from which averaged error rates are calculated. Table 2 reports on these results and, in addition, compares the four different LDAs by means of a paired $t$-test.

\section{Discussion and Conclusion}

A more sound and appealing approach to semi-supervised learning for classical LDA has been suggested. It is based on a direct optimization of the log-likelihood subject to the constraints that have been studied before in [11. It is through these constraints that unlabeled data has its influence on the final solution.

By construction, the approach using the $\Omega$ initialization will give a higher log-likelihood than the ad hoc solution. As it turns out, experimentally, the $\alpha$ initialization shows the same: it gives a log-likelihood higher than the ad hoc procedure. The resulting error rates show, however, that this does not necessarily lead to a significant performance improvement in terms of classification error 
Table 2. Results for the supervised and the three semi-supervised approaches to LDA are displayed. Underlining indicates the best performing method. Bold faced fonts indicate that these results do not differ significantly from the best result (based on a t-test).

\begin{tabular}{|c|c|c|c|c|}
\hline data set & supervised & $\begin{array}{c}\text { constrained } \\
(\text { ad hoc, [1] })\end{array}$ & $\begin{array}{c}\text { constrained } \\
(\alpha)\end{array}$ & $\begin{array}{c}\text { constrained } \\
(\Omega)\end{array}$ \\
\hline glass & .604 & .607 & .607 & .621 \\
\hline haberman & .426 & .385 & .376 & .396 \\
\hline ionosphere & .367 & .239 & .244 & .231 \\
\hline iris & .090 & .169 & .191 & $\overline{.201}$ \\
\hline parkinsons & .392 & .383 & .348 & .357 \\
\hline pendigits & .559 & .558 & .580 & .454 \\
\hline pima & .385 & .346 & .345 & .341 \\
\hline sat & .538 & .501 & .505 & .444 \\
\hline segmentation & .503 & .553 & .431 & .570 \\
\hline sonar & .440 & .358 & .350 & .344 \\
\hline spambase & .414 & .489 & .494 & .496 \\
\hline transfusion & .444 & .388 & .406 & .402 \\
\hline vowel & .730 & .744 & .768 & .715 \\
\hline wdbc & .181 & .219 & .322 & .261 \\
\hline
\end{tabular}

(cf. [12]). Therefore, even though our approach is another step in the right direction, we did not arrive at the point yet where we can guarantee reductions in expected error rates. Overall, however, the results in Table 2 at least show that the constrained approach is to be preferred over the original suggestion made in [1] when compared on the basis of classification performance.

We suspect that one of the key issues that should be studied in more depth in the future is the optimization of the constrained log-likelihood. At this point, we have little insight in how close we come to a global optimum or, generally, what would be the most effective way of reaching a satisfactory solution.

\section{References}

1. Asuncion, A., Newman, D.: UCI machine learning repository (2007), http://www.ics.uci.edu/ mlearn/MLRepository.html

2. Ben-David, S., Lu, T., Pál, D.: Does unlabeled data provably help? Worst-case analysis of the sample complexity of semi-supervised learning. In: COLT 2008, pp. 33-44 (2008)

3. Chapelle, O., Schölkopf, B., Zien, A.: Semi-Supervised Learning. MIT Press, Cambridge (2006)

4. Cohen, I., Cozman, F., Sebe, N., Cirelo, M., Huang, T.: Semisupervised learning of classifiers: Theory, algorithms, and their application to human-computer interaction. IEEE Transactions on Pattern Analysis and Machine Intelligence, 1553-1567 (2004)

5. Cozman, F., Cohen, I.: Risks of semi-supervised learning. In: Semi-Supervised Learning, ch. 4. MIT Press (2006) 
6. Dempster, A.P., Laird, N.M., Rubin, D.B.: Maximum likelihood from incomplete data via the em algorithm. Journal of the Royal Statistical Society. Series B (Methodological) 39(1), 1-38 (1977)

7. Fukunaga, K.: Introduction to Statistical Pattern Recognition. Academic Press (1990)

8. Hartley, H.O., Rao, J.N.K.: Classification and estimation in analysis of variance problems. Review of the International Statistical Institute 36(2), 141-147 (1968)

9. Liu, Q., Sung, A.H., Chen, Z., Liu, J., Huang, X., Deng, Y.: Feature selection and classification of MAQC-II breast cancer and multiple myeloma microarray gene expression data. PLoS ONE 4(12), e8250 (2009)

10. Loog, M.: Constrained Parameter Estimation for Semi-supervised Learning: The Case of the Nearest Mean Classifier. In: Balcázar, J.L., Bonchi, F., Gionis, A., Sebag, M. (eds.) ECML PKDD 2010, Part II. LNCS, vol. 6322, pp. 291-304. Springer, Heidelberg (2010)

11. Loog, M.: Semi-supervised Linear Discriminant Analysis Using Moment Constraints. In: Schwenker, F., Trentin, E. (eds.) PSL 2011. LNCS (LNAI), vol. 7081, pp. 32-41. Springer, Heidelberg (2012)

12. Loog, M., Duin, R.P.W.: The Dipping Phenomenon. In: Gimel' farb, G.L., Hancock, E., Imiya, A., Kudo, M., Kuijper, A., Omachi, S., Windeatt, T., Yamada, K. (eds.) SSPR \& SPR 2012. LNCS, vol. 7626, pp. 310-317. Springer, Heidelberg (2012)

13. Mann, G.S., McCallum, A.: Generalized expectation criteria for semi-supervised learning with weakly labeled data. The Journal of Machine Learning Research 11, 955-984 (2010)

14. McLachlan, G.J.: Iterative reclassification procedure for constructing an asymptotically optimal rule of allocation in discriminant analysis. Journal of the American Statistical Association 70(350), 365-369 (1975)

15. McLachlan, G.: Estimating the linear discriminant function from initial samples containing a small number of unclassified observations. Journal of the American Statistical Association 72(358), 403-406 (1977)

16. McLachlan, G.: Discriminant Analysis and Statistical Pattern Recognition. John Wiley \& Sons (1992)

17. McLachlan, G., Ganesalingam, S.: Updating a discriminant function on the basis of unclassified data. Communications in Statistics - Simulation and Computation 11(6), 753-767 (1982)

18. Nigam, K., McCallum, A., Thrun, S., Mitchell, T.: Learning to classify text from labeled and unlabeled documents. In: Proceedings of the Fifteenth National Conference on Artificial Intelligence, pp. 792-799 (1998)

19. Salazar, R., Roepman, P., Capella, G., Moreno, V., Simon, I., Dreezen, C., LopezDoriga, A., Santos, C., Marijnen, C., Westerga, J., et al.: Gene expression signature to improve prognosis prediction of stage II and III colorectal cancer. Journal of Clinical Oncology 29(1), 17-24 (2011)

20. Singh, A., Nowak, R., Zhu, X.: Unlabeled data: Now it helps, now it doesn't. In: Advances in Neural Information Processing Systems, vol. 21 (2008)

21. Yarowsky, D.: Unsupervised word sense disambiguation rivaling supervised methods. In: Proceedings of the 33rd Annual Meeting on Association for Computational Linguistics, pp. 189-196 (1995)

22. Zhu, X., Goldberg, A.: Introduction to Semi-Supervised Learning. Morgan \& Claypool Publishers (2009) 